\title{
Mass Transfer in Low Mass Close Binaries
}

\author{
Kinwah Wu \\ Research Centre for Theoretical Astrophysics, School of Physics, \\ University of Sydney, NSW 2006, Australia
}

\begin{abstract}
.
The mass transfer process in low mass close binaries is reviewed. The driving mechanisms and the stability properties are discussed by means of general, simple formulations. A model in terms of mass transfer instabilities is suggested to explain the outbursts of GRO J1655-40 in 1994.
\end{abstract}

\section{Introduction}

Low mass close binaries are systems consisting of a compact star and a low mass main-sequence, or evolved, companion star. As stellar winds from low mass main-sequence and subgiant stars are insignificant, mass transfer in low mass close binaries is generally caused by matter overflowing the Roche-lobe of the companion star. When matter is accreted onto the compact star, gravitational energy is released, heating the accretion matter so that it emits intense X-ray and optical radiation. Because close binaries have small orbital separations, a substantial amount of this radiation will intercept the companion star and heat up its atmosphere. The companion star therefore expands, causing more matter to overflow the Roche-lobe. In some situations, irradiative heating can lead to positive feedback, and hence alters the stability properties of the mass transfer process.

\section{Driving Forces of Mass Transfer}

\subsection{Orbital evolution}

For a system with a compact star of mass $M_{1}$ and a companion star of mass $M_{2}$, the orbital separation $a$ and the orbital angular momentum $J$ are related by $a=J^{2}\left(M_{1}+M_{2}\right) / G M_{1}^{2} M_{2}^{2}$, where $G$ is the gravitational constant. When there is mass loss, orbital angular momentum loss from the binary, or mass redistribution between two stars, the orbital separation will change. The change is given by:

$$
\frac{\dot{a}}{a}=2 \frac{\dot{J}}{J}-2 \frac{\dot{M}_{2}}{M_{2}}\left[\left(1-\frac{\beta}{q}\right)-\frac{1}{2}\left(\frac{1-\beta}{1+q}\right)-\frac{\alpha}{q}(1-\beta)(1+q)\right],
$$

where $q=M_{1} / M_{2}$ is the mass ratio, $\beta=-\dot{M}_{1} / \dot{M}_{2}$ the fraction of mass loss from the companion star being accreted onto the compact star, and $\alpha$ the 
specific angular momentum carried away by the mass loss from the system. For a semi-detached binary (i.e. the companion star fills its Roche-lobe) with $M_{2}<0.8 M_{1}$, the Roche-lobe radius of the companion star is approximately $R_{L}=0.4622 a\left[M_{2} /\left(M_{1}+M_{2}\right)\right]^{1 / 3}$ (Paczynski 1967). It follows that

$$
\frac{\dot{R}_{L}}{R_{L}}=\frac{\dot{a}}{a}+\frac{1}{3} \frac{\dot{M}_{2}}{M_{2}}\left[1-\left(\frac{1-\beta}{1+q}\right)\right] .
$$

Combining eqns (1) and (2), together with a mass-radius relation for the companion star $R_{2}=R_{\odot}\left(M_{2} / M_{\odot}\right)^{\gamma}$ (for low mass hydrogen zero-age-mainsequence stars $\gamma \approx 3 / 5$, de Loore and Doom 1992), one obtains the 'steady state' (i.e. $\dot{R}_{2}=\dot{R}_{L}$ ) mass transfer rate:

$$
\frac{\dot{M}_{2}}{M_{2}}=\frac{\dot{J}}{J}\left[\left(\frac{5}{6}+\frac{\gamma}{2}-\frac{\beta}{q}\right)-\frac{1}{3}\left(\frac{1-\beta}{1+q}\right)-\frac{\alpha}{q}(1-\beta)(1+q)\right]^{-1} .
$$

From the above equation, one can see that mass transfer can be a consequence of the orbital evolution of the binary.

For short orbital periods $\left(P_{o r b}<a\right.$ few days), orbital angular momentum can be carried away efficiently by the gravitational radiation emitted from the binary. As the orbit shrinks, the companion star is forced to overflow its Rochelobe and transfers matter to the compact star (Faulkner 1971). The typical time scale for this process is

$$
\frac{J}{\dot{J}_{G R}}=-\frac{5}{32(2 \pi)^{8 / 3}} \frac{c^{5}}{G^{5 / 3}} \frac{(1+q)^{1 / 3}}{q} \frac{P_{o r b}^{8 / 3}}{M_{2}^{5 / 3}},
$$

where $c$ is the speed of light. For binaries with $M_{1}=0.7 M_{\odot}, M_{2}=0.35 M_{\odot}$, and $P_{\text {orb }}=0.17$ day (the typical parameters for cataclysmic variables) $J / \dot{J}_{G R} \sim$ $1.4 \times 10^{10} \mathrm{yr}$. The corresponding mass transfer rate is $\sim 3 \times 10^{-11} M_{\odot} / \mathrm{yr}$. Many cataclysmic variables are, however, found to have mass transfer rates significantly higher than this value (see Warner 1987), implying the presence of other more efficient driving mechanisms.

It was proposed that magnetic braking of the companion star, through tidal coupling with the orbital motion, can result in orbital angular momentum loss (Verbunt and Zwaan 1981). Calculations, in which magnetic braking models for low mass cool single stars are adopted and parametrised for binaries via a certain scaling law (e.g. Skumanich 1972), showed that the magnetic braking process is indeed efficient, and its typical time scale is

$$
\frac{J}{\dot{J}_{M B}}=-\frac{2 \times 10^{28}}{(2 \pi)^{10 / 3}} \frac{f^{2} G^{2 / 3}}{k^{2}} \frac{M_{\odot}^{4 \gamma}}{R_{\odot}^{4}} \frac{q}{(1+q)^{1 / 3}} M_{2}^{(2-12 \gamma) / 3} P^{10 / 3} \mathrm{sec},
$$

where $k^{2}=0.1$ for cool low mass star, and $f^{2} \sim 1$, depending on the assumed scaling law. All quantities in the above equation are in c.g.s units. In deriving the magnetic braking time scale, the mass-radius relation $R_{2} \propto M_{2}^{\gamma}$ and the braking law given in Verbunt and Zwaan (1981) are used. $J / \dot{J}_{M B}$ is about $10^{8}$ yr for $M_{1}=0.7 M_{\odot}, M_{2}=0.35 M_{\odot}$, and $P_{\text {orb }}=0.17$ day. The mass 
transfer rate is $\sim 4 \times 10^{-9} M_{\odot} / \mathrm{yr}$, which is significantly higher than the rate of mass transfer driven by gravitational radiation. With additional assumptions the magnetic braking models can also explain observations such as the period gap of cataclysmic variables (Rappaport, Verbunt and Joss 1983; Spruit and Ritter 1983). Nevertheless, there are still many difficulties and uncertainties, both theoretical and observational, in the magnetic braking models (see Warner 1987; $\mathrm{Li}, \mathrm{Wu}$ and Wickramasinghe 1994).

\subsection{Nuclear evolution}

In deriving eqn (3), the mass-radius relation $R_{2} \propto M_{2}^{\gamma}$ (with $\gamma$ approximately constant) is assumed to be valid throughout the mass transfer process. This assumption generally holds for systems with a low mass main-sequence companion star, as the time which the companion star spends in its main-sequence phase is much longer than the time scale for the orbital evolution of the close binary. For a subgiant companion star, its evolution time scale can be short enough that the $\dot{J} / J$ term in eqn (1) can be neglected. Then, combining eqns (1) and (2), one obtains

$$
\frac{\dot{M}_{2}}{M_{2}}=-\frac{1}{2} \frac{\dot{R}_{2}}{R_{2}}\left[\left(\frac{5}{6}-\frac{\beta}{q}\right)-\frac{1}{3}\left(\frac{1-\beta}{1+q}\right)-\frac{\alpha}{q}(1-\beta)(1+q)\right]^{-1} .
$$

The above equation implies that mass transfer can be driven by the expansion of the radius of the companion star.

\subsection{Irradiative heating}

In the presence of irradiative heating, the surface temperature of the companion star is not a constant. Therefore, it needs to be treated as an explicit variable in the formulation. Generally, the rate of mass outflow from the Roche-lobe of the companion star is

$$
-\dot{M}_{2} \propto T_{2}^{3 / 2} \exp \left[-\lambda \frac{\left(R_{L}-R_{2}\right)}{T_{2}^{1 / 2}}\right]
$$

(see Lubow and Shu 1975; Ritter 1995), where $T_{2}$ is the effective temperature of the companion star near the inner Lagrangian point, and $\lambda$ is a slow varying parameter depending on the spectral type of the companion star and the mass ratio of the stars in the binary. The variation of $R_{2}$ is given by

$$
\frac{\dot{R}_{2}}{R_{2}}=\frac{\partial}{\partial t} \ln R_{2}+\zeta_{a d} \frac{\dot{M}_{2}}{M_{2}}+\left(\frac{\partial \ln R_{2}}{\partial \ln \epsilon}\right) \frac{\dot{\epsilon}}{\epsilon},
$$

where $\zeta_{a d}$ is the adiabatic index of the star, and $\epsilon$ is energy deposited onto the companion star by irradiation. Similarly, the variation of $R_{L}$ is

$$
\frac{\dot{R}_{L}}{R_{L}}=\frac{\partial}{\partial t} \ln R_{L}+\zeta_{J} \frac{\dot{M}_{2}}{M_{2}}
$$

where $\zeta_{J}$ specifies the change in the Roche-lobe radius of the companion star due to mass transfer. It is not straight forward to obtain an expression for the 
effective temperature $T_{2}$. However, one can argue from the energy-balance point of the view that $T_{2} \approx\left[\left(F_{i r r}\left(\dot{M}_{2}\right)+F_{2}\right) / 4 \sigma\right]^{1 / 4}$, where $F_{i r r}\left(\dot{M}_{2}\right)$ is the irradiation flux, $F_{2}$ the intrinsic flux of the companion star, and $\sigma$ the Stefan-Boltzmann constant.

\section{Stability Properties of Mass Transfer with Irradiative heating}

In the absence of irradiative heating, eqn (3) shows that steady mass transfer can be driven by orbital angular evolution if the [.....] term in the equation is positive. For $\beta=1$ and $\alpha=0$, the criterion for steady mass transfer is $q>6 /(5+3 \gamma)$. This criterion is easily satisfied for systems with a low mass companion star. For systems with a mass ratio smaller than the critical value, the shrinking of the orbital separation is not slow enough to ensure $\left|\dot{R}_{L}\right| \ngtr\left|\dot{R}_{2}\right|$. (The critical mass ratio for the general situation (i.e. $\beta<1$ and $\alpha>0$ ) can also be derived from eqn (1) by solving $q$ for $[\ldots .]>$.0 .)

Mass transfer driven by nuclear evolution of the companion star is generally stable for low-mass binaries. The mass redistribution merely causes the orbital separation to increase gradually.

In deriving eqns (3) and (6), feedback effects due to irradiative heating of the companion star are not considered. Studies (e.g. Osaki 1985; Hameury, King and Lasota 1986; Kovetz, Prialnik and Shara 1988; Sarna 1990; Harpaz and Rappaport 1991, 1994; King et al. 1995; Ritter 1995; Wu, Wickramasinghe and Warner 1995) have shown that irradiative heating not only modifies the mass transfer rate substantially but also changes the stability properties of the mass transfer process. Although a star will certainly expand when it is irradiatively heated, the actual response will depend on details, such as the energy spectrum of the irradiation, the spectral type of the star and even the history of the irradiation process.

When a star is irradiated, a radiative layer will develop at the top of its originally convective envelope. The newly formed radiative layer shields the star from irradiation, and prevents further heating. As a consequence, a new equilibrium stellar structure will result. Studies of irradiative heating in lowmass X-ray binaries have shown that an irradiatively heated companion star can gradually establish an equilibrium structure. After the new equilibrium is reached, the mass transfer process is then driven by the orbital evolution of the binary (Harpaz and Rappaport 1991).

As irradiative heating suppresses convection in the stellar envelope, it has a larger effect on low mass main-sequence stars than on high mass stars (Podsiadlowski 1991). It is therefore natural to argue that shorter period binaries should exhibit stronger feedback under irradiative heating, and the corresponding mass transfer process is more likely to be unstable. Although some studies on cataclysmic variables (e.g. Osaki 1985; Sarna 1990; Wu, Wickramasinghe and Warner 1995) showed results consistent with this argument, other studies (e.g. King et al. 1995) seemed to arrive at the opposite conclusion. This dispute has not yet been settled. However, there is further concern that other factors, such as meridional circulation (Sarna 1990) or shielding of the radiation by an accretion disk, can complicate the situation. 
Mass transfer driven by nuclear evolution in the presence of irradiative heating is less complicated than that driven by orbital evolution. As an increase in the mass transfer rate leads to an increase in the radius of the companion star, one can conclude from eqn (6) that irradiative heating tends to destabilise the mass transfer process. The mass transfer rate will continue to increase after the onset of the instability. Only when the whole envelope of the evolved star is consumed, or the feedback cycle is disrupted (e.g. by the formation of a geometrically thick accretion disk which shields the radiation emitted from the accreting compact star), does mass transfer cease.

\section{Outbursts of GRO J1655-40 in 1994}

The X-ray transient GRO J1655-40 is a candidate black hole binary with a deduced mass for its compact star of $4.0-5.2 M_{\odot}$ (Bailyn et al. 1995a). The companion star is a F5 subgiant star (Bailyn et al. 1995b) and is believed to fill its Roche-lobe. The 2.62 day orbital period of GRO J1655-40 implies an orbital separation of about $14 R_{\odot}$ and a Roche-radius of $\approx 3.7 R_{\odot}$ for the companion star, assuming the masses of the black hole and the subgiant star to be $4.5 M_{\odot}$ and $1.0 M_{\odot}$ respectively.

The evolution calculations of Maeder and Meynet (1988) showed that the rate of radius expansion, $\dot{R}_{2} / R_{2}$, is about $3 \times 10^{-9} /$ yr for a $1.0 M_{\odot}$ evolved Population I star with a radius of $3.7 R_{\odot}$. With $\dot{R}_{2} / R_{2}$ known, the mass transfer rate due to the nuclear evolution is estimated from eqn (6), and its value is $\sim 4.0 \times 10^{-10} M_{\odot} / \mathrm{yr}$. Given that the energy liberated by accretion onto a black hole is less than $6 \%$ of the rest mass energy of the accretion matter, the accretion luminosity should be less $1.4 \times 10^{36} \mathrm{erg} \mathrm{s}^{-1}$.

Since its discovery in 1994 (Harmon et al. 1995), GRO J1655-40 has continued to show X-ray outbursts. This implies large variations in the rate of accretion onto the black hole. The common interpretation is that instabilities have developed in the accretion disk and caused the accretion disk to collapse, emptying the accretion matter into the black hole. Although the first two outbursts of GRO J1655-40 may have been due to accretion disk instabilities, it is difficult to understand how the even stronger third outburst (JD 2,449,655) could occur if most of the accretion matter in the accretion disk had already been drained in the two previous outbursts.

We suggest that the third outburst of GRO J1655-40 in 1994 (see the Xray light curve shown in $\mathrm{Wu}$ and Hunstead, this volume) was due to irradiative heating induced mass transfer instabilities. The intense X-rays produced during the first two outbursts would inevitably have intercepted the companion star, heating its envelope. As the companion star is an evolved subgiant star, the mass transfer became unstable in the presence of strong irradiative heating. Thus, a huge third outburst was triggered.

\section{Conclusions}

Stable mass transfer can occur in low mass close binaries when it is driven by the orbital evolution of binaries or the nuclear evolution of the companion star. The 
radiation resulting from mass transfer can irradiatively heat the companion star, causing positive feedback in the mass transfer process. As a result, the mass transfer process may be destabilised. We suggest that the third outburst in GRO J1655-40 may be due to mass transfer instabilities caused by irradiative heating of the companion star during the previous outbursts. If the mass transfer of GRO $\mathrm{J} 1655-40$ is driven by nuclear evolution, the accretion luminosity in quiescence would be less than $\sim 1.4 \times 10^{36} \mathrm{erg} \mathrm{s}^{-1}$.

The author thanks M. Burgess, R. W. Hunstead and D. T. Wickramasinghe for discussions and A. F. Tennant and E. Rowe for comments on the manuscript.

\section{References}

Bailyn, C. D. 1995a, Nature, 374, 701

Bailyn, C. D. 1995b, IAUC No. 6173

de Loore C. W. H. and Doom, C. 1992, Structure and Evolution of Single and Binary Stars, Dordrecht: Kluwer

Faulkner, J. 1971, ApJ, 170, L99

Hameury, J. M., King, A. R. and Lasota, J. P. 1986, A\&A, 162, 71

Harmon, B. A. et al. 1995, Nature, 374, 303

Harpaz, A. and Rappaport, S. 1991, ApJ, 383, 739

Harpaz, A. and Rappaport, S. 1994, ApJ, 434, 283

King, A. R. et al. 1995, ApJ, 444, L37

Kovetz, A., Prialnik, D. and Shara, M. M. 1988, ApJ, 325, 828

Li, J., Wu, K. and Wickramasinghe, D. T. 1994, MNRAS, 268, 61

Lubow, S. H. and Shu, F. H. 1975, ApJ, 198, 447

Maeder, A. and Meynet, G. 1988, A\&AS, 123, 33

Osaki, Y. 1985, A\&A, 144, 369

Paczynski, B. 1967, Acta Astron., 17, 287

Podsiadlowski, Ph. 1991, Nature, 350, 136

Rappaport, S., Verbunt, F. and Joss, P. C. 1983, ApJ, 275, 713

Ritter, H. 1995, in Evolutionary Processes in Binaries Stars, R. A. M. J. Wijers et al., Dordrecht: Kluwer, 223

Sarna, M. J. 1990, A\&A, 239, 163

Skumanich, A. 1972, ApJ, 171, 565

Spruit, H. C. and Ritter, H. 1983, A\&A, 124, 267

Verbunt, F. and Zwaan, C. 1981, A\&A, 100, L7

Warner, B. 1987, MNRAS, 227, 23

Wu, K., Wickramasinghe, D. T. and Warner, B. 1995, in Cataclysmic Variables, B. Bianchini et al., Dordrecht: Kluwer, 315 